\title{
A Study on a Gain-Enhanced Antenna for Energy Harvesting using Adaptive Particle Swarm Optimization
}

\author{
Seong-In Kang*, Koon-Tae Kim*, Seung-Jae Lee*, Jeong-Phill Kim*, Kyung Choi** \\ and Hyeong-Seok Kim ${ }^{\dagger}$
}

\begin{abstract}
In this paper, the adaptive particle swarm optimization (APSO) algorithm is employed to design a gain-enhanced antenna with a reflector for energy harvesting. We placed the reflector below the main radiating element. Its back-radiated field is reflected and added to the forward radiated field, which could increase the antenna gain. We adopt the adaptive particle swarm optimization (APSO) algorithm, which improves the speed of convergence with a high frequency solver. The result shows that performance of the optimized design successfully satisfied the design goal of the frequency band, gain and axial ratio.
\end{abstract}

Keywords: Energy harvesting, Reflector, Gain-enhanced antenna, Adaptive particle swarm optimization, Circular polarization

\section{Introduction}

Recently, the field of energy harvesting is drawing social and industrial attention, since energy is running out. It should be recycled and consumed efficiently. Especially, the infrastructure needs to be operated intelligently. Sensor nodes and their link play an important role in monitoring and maintaining the quality of energy such as electric power. The sensor node is an electronic device that consumes the electric power for tasking, and its battery should be substituted on a regular basis [1-3]. Changing the battery of a sensor node is difficult job, because it is placed high above the ground or a high wall. Therefore, Wireless charging technology using RF energy harvesting is recommended in this case.

Energy harvesting is to collect radio frequency (RF) energy propagating in the air. The ambient RF power is a good potential candidate for the energy supply as it is widely emitted from numerous reliable electromagnetic power resources $[4,5]$. An energy harvesting equipment always needs one antenna or more as a core component which can catch the ambient RF power, when the optimal condition is met. For efficient energy harvesting, a good antenna with a circular polarization (CP), low profile and high gain is required.

$[6,7]$. Regarding the necessity of the circular polarization, the RF energy flies in the air with an arbitrary polarization, and the circular polarization will increase the probability of catching the incoming energy [8].

$\dagger$ Corresponding Author: School of Electrical and Electronics Eng., Chung-Ang Univ., Korea. (kimcaf2@cau.ac.kr)

* School of Electrical and Electronics Eng., Chung-Ang Univ., Korea. (dolf104@cau.ac.kr)

** Dept. of Electronics Eng., Kangwon National University, Korea. (kyunchoi@kangwon.ac.kr)

Received: December 31, 2014; Accepted: March 13, 2015
Considering the easy installation of a sensor node on a high wall, a small area of structure is preferred, which will make a layout complicated and its optimization design time-consuming. To overcome the degraded gain caused by the installation on the wall at its back, its antenna-gain should be enhanced. When the reflector is placed below the main radiating element, its back-radiated field is reflected and added to the forward radiated field, which will possibly increase the antenna gain [9].

As is mentioned above, the optimization of the energy harvesting is a complicated problem, Because a small structure are more complicated and has more design parameters and it should overcome its originally low gain and low axial ratio(AR) of the circular polarization. So, we use and adaptive particle swarm optimization (APSO) algorithm to optimize the antenna design with numerous design parameters [10]. The APSO has a faster convergence in the process than the PSO which we are going to show in the paper.

The APSO algorithm is implemented in visual basic (VB), and connected to HFSS for finite element analysis through the script function to optimize the antenna in an accurate way [11]. The proposed design is also tested and verified to have the desired performance through simulations and experiments.

\section{A Gain-Enhanced Antenna with Reflector}

The efficiency of RF energy harvesting is estimated as the received RF power. According to the friis transmission formula, the received RF power at the antenna can be expressed as 


$$
P_{r}=P_{t} G_{t}\left(\frac{\lambda}{4 \pi R}\right)^{2} G_{r}
$$

where, $P_{r}$ is received power, $P_{t}$ is transmitted power, $G_{1}$ is transmitted antenna gain, $G_{r}$ is received antenna gain, $R$ is distance between transmitted and received antenna. Therefore, the received antenna with a high gain is a key factor to high received power. One of the ways to enhance the gain of the planar radiating element is to let the field interfere with each other. To do this, we suggest the geometry as in Fig. 1. It is composed of the ring-slot antenna backed by a reflector such as Fig. 1 (b).

The ring-slot patch antenna is operated in $\mathrm{TM}_{11}$ mode. Thus the circumference of the ring is the half wavelength of the operating frequency. Complying with the need to reduce the size of antenna, we insert slits as shown in Fig. 1 (a) to make the effective length of the current path along the ring increase. As an additional design element, the patch corners are cut to create a circular polarization for better reception of energy in an arbitrary angle position [12].

This circular polarized field is radiated forward and backward together. The backward wave hits the reflector and is reflected into the forward direction. With the distance between the radiating element and the reflector, the characteristics of the interference between the forward and reflected field change and can lower the gain. So, the optimal distance should be met. To explain the interference effect, Fig. 2 shows the radiation patterns with and without the reflector.

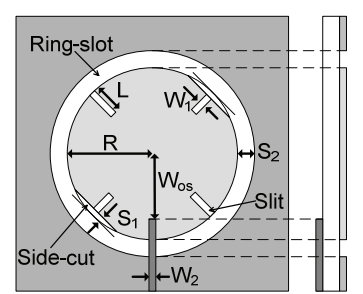

(a)

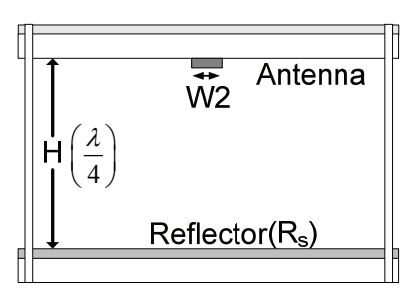

(b)
Fig. 1. The reflector ring-slot patch antenna with reflector and design parameters: (a) top; (b) side.

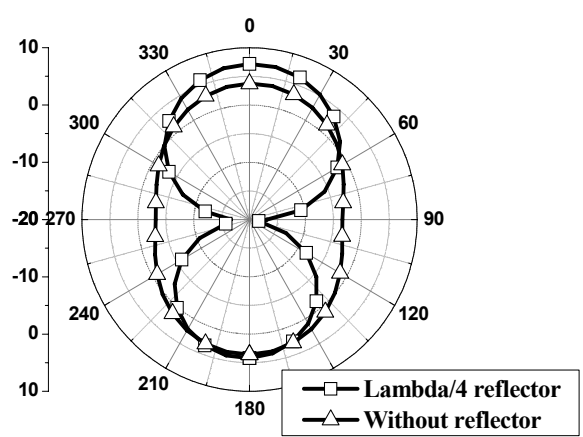

Fig. 2. The radiation pattern of antenna with and without reflector.

\section{Optimal Design of Antenna}

\subsection{Implementation of APSO algorithm}

To find the optimal distance between the radiating element and reflector, we adopt the adaptive PSO (APSO). The APSO is a real-time evolutionary four state estimation procedure. Four state including exploration, exploitation, convergence, jumping-out in each iteration [10]. The flow chart of APSO algorithm shows in Fig. 3.

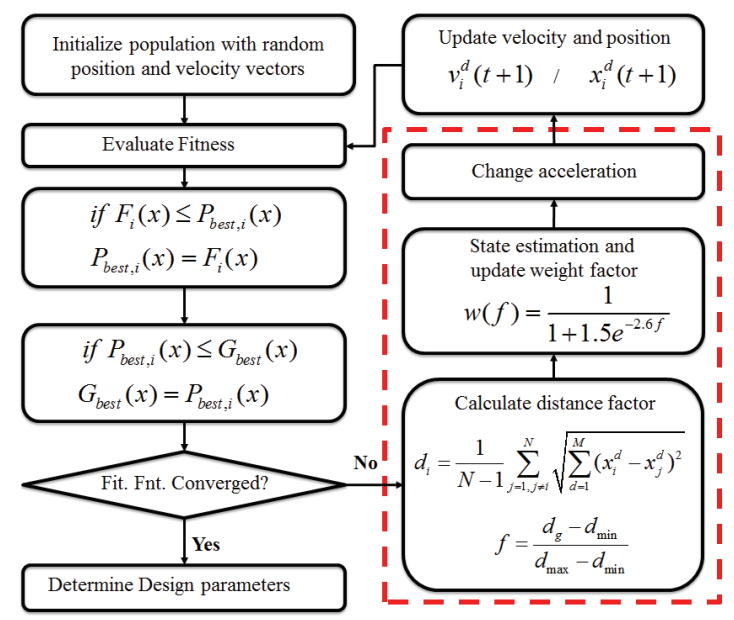

Fig. 3. The Flow chart of the APSO algorithm.

\subsubsection{Calculate distance factor}

At the current position, the mean distance of each particle $i$ is calculated to all the other particles. For example, this mean distance can be measured using an Euclidian metric

$$
d_{i}=\frac{1}{N-1} \sum_{j=1, j \neq i}^{N} \sqrt{\sum_{d=1}^{M}\left(x_{i}^{d}-x_{j}^{d}\right)^{2}}
$$

where $N$ and $M$ are the population size and the number of dimensions, respectively.

Denote $d_{i}$ of the globally best particle as $d_{g}$. Compare all $d_{i}$ 's, and determine the maximum and minimum distances $d_{\max }$ and $d_{\min }$. Compute an "evolutionary factor" $f$ as defined by

$$
f=\frac{d_{g}-d_{\text {min }}}{d_{\text {max }}-d_{\text {min }}} \in[0,1]
$$

\subsubsection{State estimation and update weight factor}

Classify $f$ into one of the four sets $S_{1}, S_{2}, S_{3}$, and $S_{4}$, which represent the states of exploration, exploitation, convergence, and jumping out, respectively. The key to fuzzy classification is overlap memberships. The formulation for numerical implementation of the classification is as follows. 
Exploration : A medium to large value of $f$ represents $S_{1}$,

Exploitation : A shrunk value of $f$ represents $S_{2}$,

Convergence : A minimal value of $f$ represents $S_{3}$,

Jumping-out : When PSO is jumping out of a local optimum. The largest value of $f$ reflect $S_{4}$,

It would be beneficial to allow $w$ to follow the evolutionary states using a sigmoid mapping $w(f)$

$$
\omega(f)=\frac{1}{1+1.5 e^{-2.6 f}} \quad \forall f \in[0,1]
$$

\subsubsection{Change acceleration}

Parameter c1 represents the "self-cognition" that pulls the particle to its own historical best position. Parameter $c_{2}$ represents the "social influence" that pushes the swarm to convergence to the current globally best region.

Strategy 1 - increasing $c_{1}$ and decreasing $c_{2}$

Strategy 2 - increasing $c_{1}$ slightly and decreasing $c_{2}$ slightly Strategy 3 - increasing $c_{1}$ slightly and increasing $c_{2}$ slightly Strategy 4- decreasing $c_{1}$ and increasing $c_{2}$

\subsubsection{Update velocity and position}

The velocity of the particle is changed according to the relative locations of $P_{\text {best }}$ and $G_{\text {best }}$. It is accelerated in the directions of these locations of greatest fitness according to the following equation:

$$
\begin{aligned}
v_{i}^{d}(t+1)= & w(f) \times v_{i}^{d}(t)+c_{1} \times r_{1} \times\left(P_{\text {best }, i}(t)-x_{i}^{d}(t)\right) \\
& +c_{2} \times r_{1} \times\left(g_{\text {best }, i}(t)-x_{i}^{d}(t)\right)
\end{aligned}
$$

where $v_{i}^{d}(t)$ is the velocity of the particle and $x_{i}^{d}(t)$ is the particle's coordinate in the $m$ dimension and $w$ is the inertial weight. $c_{1}$ and $c_{2}$ are scaling factors of stochastic probability that determine the relative attraction to $P_{\text {best }}$ and $G_{\text {best }} . r_{1}$ and $r_{2}$ are random variables whose value is provided between 0 and 1 . The new position of each particle is updated according to the linear searching with an arbitrary interval.

\subsection{Design goal and parameter of the antenna}

We implemented the APSO program for the antenna problem such that the dimension of the system is 9 which is the number of the design parameters, and the number of particles is 15. For the part of Finite Element Analysis of the model, the HFSS is used [12]. The APSO is written in Visual Basic that is linked to the HFSS through the script function of the HFSS.

The design goal is to obtain a global optimum for making the antenna's resonant frequency at $2.45 \mathrm{GHz}, \mathrm{CP}$, enhanced gain, and suppressing the harmonics radiation.

To improve the antenna gain, we minimize back-radiated field and maximize forward radiated field. So, main lobe level of the radiation pattern is upper $7 \mathrm{dBi}$ and back lobe level is lower $0 \mathrm{dBi}$. Also, the $\mathrm{S} 11$ of resonant band (2.2 $2.6 \mathrm{GHz})$ and stop band $(3 \sim 8 \mathrm{GHz})$ is below $-12 \mathrm{~dB}$ and above $-8 \mathrm{~dB}$, respectively. To realize circular polarization, the AR should be less than $3 \mathrm{~dB}$ in resonant frequency.

The fitness function of the APSO with constraints on the resonance frequency, return loss, and gain is defined as;

$$
F_{t}(x)=\alpha_{1} F_{1}(x)+\alpha_{2} F_{2}(x)+\beta_{1} G_{1}(x)+\beta_{2} G_{2}(x)+A_{f}(x)
$$

where,

$$
\begin{aligned}
& F_{1}(x)=\int_{f_{1}}^{f_{2}}\left(s\left(x_{i}\right)-s_{1}\right)^{2} d f \quad \text { if } s>s_{1} \\
& F_{2}(x)=\int_{f_{3}}^{f_{4}}\left(s\left(x_{i}\right)-s_{2}\right)^{2} d f \quad \text { if } s>s_{2} \\
& G_{1}(x)=\int_{\theta_{1}}^{\theta_{2}}\left(g\left(\theta, f_{2.45 G H z}\right)-g_{1}\right)^{2} d \theta \text { if } g<g_{1} \\
& G_{2}(x)=\int_{\theta_{3}}^{\theta_{4}}\left(g\left(\theta, f_{2.45 G H z}\right)-g_{2}\right)^{2} d \theta \text { if } g>g_{2} \\
& A_{f}(x)=\left.\left(a\left(x_{i}\right)-a_{t}\right)^{2}\right|_{f=2.45 G H z} \text { if } a_{i}>a_{t}
\end{aligned}
$$

Functions $F_{12}, G_{12}$, and $A_{f}$ represent the resonant frequency, gain and axial ratio, respectively. $s_{12}, g_{12}$ and $a_{t}$ denote the levels of return loss, the gain and the axial ratio of designated resonant frequency, respectively. We set the resonant frequency band from $f_{1}=2.2 \mathrm{GHz}$, to $f_{2}=2.6 \mathrm{GHz}$, and harmonic supersession band from $f_{3}=3.0 \mathrm{GHz}$ to $f_{4}=$ 8.0GHz. Main lobe of the radiation is set from $\theta_{1}=-20 \mathrm{deg}$ to $\theta_{2}=20 \mathrm{deg}$, and back lobe from $\theta_{1}=-160 \mathrm{deg}$ to $\theta_{2}=$ $160 \mathrm{deg}$. Upper and lower bound target values are $s_{1}=-12$ $\mathrm{dB}, s_{2}=-8 \mathrm{~dB}, g_{1}=7 \mathrm{dBi}, g_{2}=0 \mathrm{dBi}$, respectively. Weight $\alpha_{1}$, $\alpha_{2}, \beta_{1}, \beta_{2}$ are the inverse values of the number of sampling points.

Some calculated values in the function which satisfied the requirement in the earlier stage may produce too large a value to the whole function. It should be adjusted to a proper value in minimizing the objective function. As a numerical manipulation, putting the function value 0 when it satisfied the required limit as (6) is much helpful.

\subsection{Optimal design result}

Initial and Optimal parameters are shown in Table 1 and the convergence of fitness function is illustrated in Fig. 4. It can be found that the band-basis objective function works very well for both the resonance and suppression bands and perform a global search with faster convergence

Table 1. The values of design parameters in antenna [mm]

\begin{tabular}{c|c|c|c|c|c|c|c|c|c}
\hline State & $\mathrm{W}_{\mathrm{OS}}$ & $\mathrm{R}$ & $\mathrm{S}_{1}$ & $\mathrm{~S}_{2}$ & $\mathrm{~L}$ & $\mathrm{~W}_{1}$ & $\mathrm{~W}_{2}$ & $\mathrm{H}$ & $\mathrm{R}_{\mathrm{s}}$ \\
\hline Initial & 1.71 & 14.46 & 2.62 & 0.53 & 3 & 1.53 & 1 & 31 & 50 \\
\hline Max & 4 & 16 & 3 & 1.5 & 8 & 2.5 & 2 & 33 & 70 \\
\hline Min & 1 & 13 & 1 & 0.3 & 2 & 1 & 1 & 29 & 50 \\
\hline Optimal & 1 & 15.91 & 3 & 0.89 & 2 & 1 & 1 & 29 & 50 \\
\hline
\end{tabular}


speed than PSO algorithm. The optimal results of APSO after 57 iterations are presented in Fig. 4 but the PSO algorithm not convergence.

The variations of the characteristics of the antenna are shown in Fig. 5 7. The optimal result of return loss is -12 $\mathrm{dB}$ under at resonant frequency band, $\mathrm{AR}$ goes to $1.91 \mathrm{~dB}$

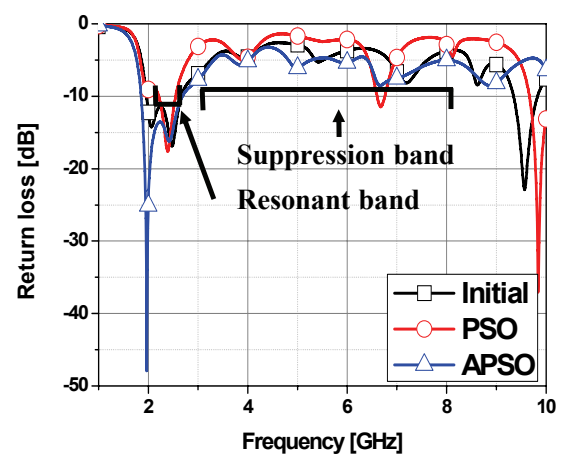

Fig. 4. The return loss distribution after optimization.

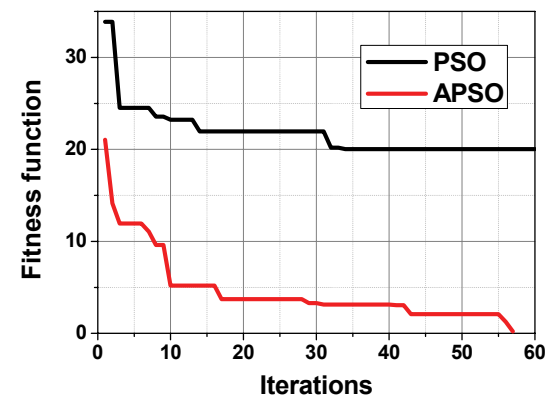

Fig. 5. Convergence of the fitness function APSO and PSO.

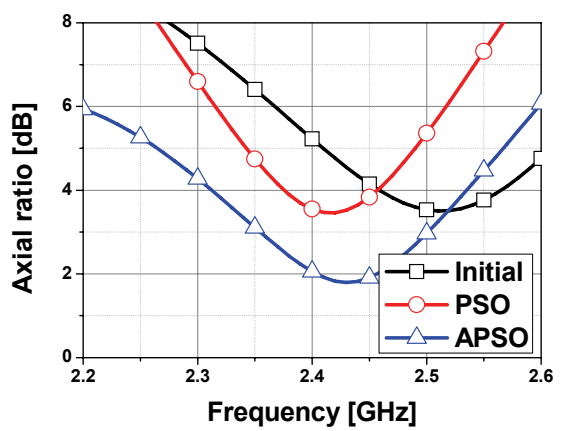

Fig. 6. The axial ratio after optimization.

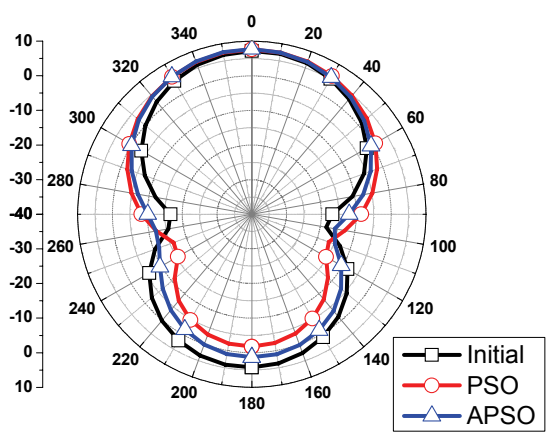

Fig. 7. The radiation pattern after optimization. on the $2.45 \mathrm{GHz}$, and gain of antenna has main lobe 7.69 $\mathrm{dBi}$, back lobe $1.32 \mathrm{dBi}$, which can be proved that it reaches the proposed design goal.

\section{Manufacturing and Measurement}

The proposed design is fabricated on FR-4 substrate and measured as shown in Fig. 8 9. The predicted and the measured return loss are compared in Fig. 8, where the design goals are denoted with s1, and s2. The measured bandwidth is from $2.2 \sim 2.6 \mathrm{GHz}$ and $3 \sim 8 \mathrm{GHz}$, which shows a good agreement with the simulation results and the design goal.

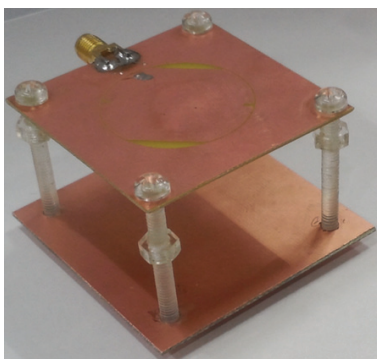

Fig. 8. The fabrication of the proposed reflector antenna.

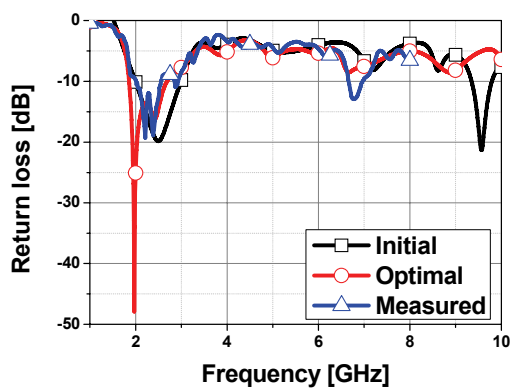

Fig. 9. Comparison of the measured and the simulated return loss for optimal design.

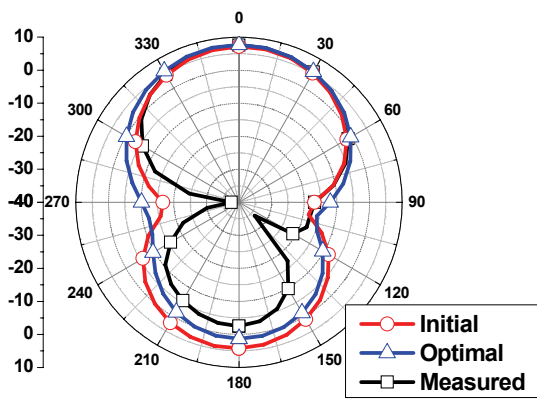

Fig. 10. Comparison of the measured and the simulated radiation pattern for optimal design.

\section{Conclusion}

A circular polarized antenna with enhanced gain for energy harvesting was designed. We placed the reflector 
below the main radiating element and its back-radiated field is reflected and added to the forward radiated field, which could increase the antenna gain.

We adopted the adaptive particle swarm optimization (APSO) algorithm, which improves the speed of convergence with a high frequency solver. The analysis reveal that the fitness function converges very well with APSO optimization algorithm and the performance of the optimized design successfully satisfied the design goal of the frequency band, gain and axial ratio.

The proposed optimized antenna was fabricated. We showed the simulated \& measured results to verify the performance of the antenna. We expect that the optimized antenna can be used for a variety of applications.

\section{Acknowledgement}

This research was supported by Basic Science Research Program through the National Research Foundation of Korea (NRF) funded by the Ministry of Education, Science and Technology (2012R1A1A2007758) and in part by the Chung-Ang University Excellent Student Scholarship in 2014.

\section{References}

[1] Z. Popovic, E.A. Falkenstein, D. Costinett, R. Zane, "Low-Power Far-Field Wireless Powering for Wireless Sensors", Proceedings of the IEEE, Vol. 101, No. 6, pp. 1397-1409, 2013.

[2] Z. Popovic, S. Korhummel, S. Dunbar, R. Scheeler, A. Dolgov, R. Zane, E. Falkenstein, J. Hagerty, "Scalable RF Energy Harvesting", IEEE Transactions on Microwave Theory and Techniques, Vol. 62, No. 4, pp. 1046-1056, 2014.

[3] Erez Falkenstein, Michael Roberg, Z. Popovic, "LowPower Wireless Power Delivery", IEEE Transactions on Microwave Theory and Techniques, Vol. 60, No. 7, pp. 2277-2286, 2012.

[4] Hucheng Sun, Yong-Xin Guo, Miao He, Zheng Zhong, "Design of a High-Efficiency 2.45-GHz Rectenna for Low-Input-Power Energy Harvesting”, IEEE Antennas and Wireless Propagation Letters, Vol. 11, pp. 929932, 2012.

[5] Hucheng Sun, Yong-Xin Guo, Miao He, Zheng Zhong, "A Dual-Band Rectenna Using Broadband Yagi Antenna Array for Ambient RF Power Harvesting", IEEE Antennas and Wireless Propagation Letters, Vol. 12, pp. 918-921, 2013.

[6] U. Olgun, C.-C. Chen, J.L. Volakis, "Design of an efficient ambient WiFi energy harvesting system", IET Microwaves, Antennas \& Propagation, Vol. 6, No. 11, pp. 1200-1206, 2012.

[7] J.A. Hagerty, F.B. Helmbrecht, W.H. McCalpin, R.
Zane, Z.B. Popovic, "Recycling ambient microwave energy with broad-band rectenna arrays", IEEE Transactions on Microwave Theory and Techniques, Vol. 52, No. 3, pp. 1014-1024, 2004.

[8] Yu-Jiun Ren and Kai Chang, "5.8-GHz Circularly Polarized Dual-Diode Rectenna and Rectenna Array for Microwave Power Transmission", IEEE Transaction on Microwave Theory and Techniques, Vol. 54, No. 4, pp. 1495-1502, 2006.

[9] Y. Ranga, K.P. Esselle, L. Matekovits, S.G. Hay, "Increasing the gain of a semicircular slot UWB antenna using an FSS reflector", 2012 IEEE-APS Topical Conference on Antennas and Propagation in Wireless Communications (APWC), pp. 478-481, 2012.

[10] Zhi-Hui Zhan, Jun Zhang, Yun Li, H.S.-H. Chung, "Adaptive Particle Swarm Optimization", IEEE Transactions on Systems, Man, and Cybernetics, Part B: Cybernetics, Vol. 39, No. 6, pp. 1362-1381, 2009.

[11] HFSS, Version 13, Ansoft Corporation LLC, 225 West Station, Square Suite 200, Pittsburgh, PA 15219-1119, 2011.

[12] Koon-Tae Kim ; Jeong-Hyeok Lee ; Kyung Choi ; Tae-Kyung Chung ; Hyeong-Seok Kim “An Optimal Design of Compact Ring-Slot Antenna for a Rectenna System With Numerical Manipulation", IEEE Transactions on Magnetics, Vol. 50, No. 2, 2014.

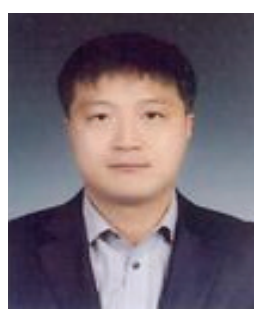

Seong-In Kang was born in Jin-ju, South Korea. He received his B.S. degree in electrical engineering from Chang-Won National University and M.S. degree in Information and Communication from Chung-Ang University, South Korea in 2008 and 2010, respectively. He is working toward the $\mathrm{Ph} . \mathrm{D}$ degree in electrical and electronics engineering at Chung-Ang University, South Korea. His interests include optimize design of passive component, equivalent circuit model, analysis on RF components and wireless power transfer.

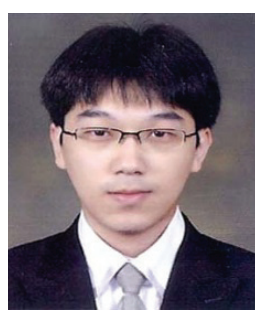

Koon-Tae Kim was born in Jeju, South Korea. He received his B.S. degree in Information Telecommunication from Seo-Kyeong University and M.S. and Ph.D. degrees in electrical and electronics engineering from Chung-Ang University, South Korea in 2008, 2010 and 2014, respectively. His interests include optimize design of passive component, equivalent circuit model and analysis on RF components 


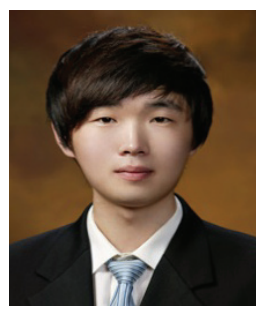

Seung-Je Lee was born in Jeollanam do, South Korea in 1988. He received his B.S. degree in electrical and electronics engineering from Chung-Ang University, South Korea in 2014. He is working toward the M.S. degree in electrical and electronics engineering at the same university. His interests include analysis of RF components, antennas.

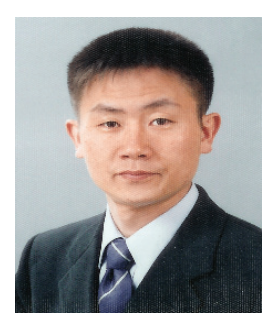

Jeong-Phill Kim was born in Jeju, South Korea. He received his B.S in electrical engineering from Seoul National University and M.S and Ph.D. degrees in electrical engineering from Pohang University of Science and Technology (POSTECH), South Korea in 1988, 1900 and 1998, respectively. From 1990 to 2001, he worked at LIG Nex1 and from 2001 to now, he has been a professor of School of Electrical and Electronics Engineering, Chung-Ang University, Seoul, South Korea. His current research interest include microstrip array antenna, phased array and digital beamforming, radar system design and signal processing and random noise radar.

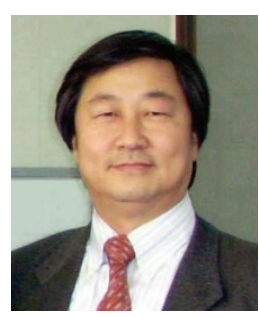

Kyung Choi was born in Taegu, South Korea in 1958. He received his B.S., M.S., and Ph.D. degrees in electrical engineering from Seoul National University, South Korea in 1981, 1983, and 1988, respectively. Since 1989, he has been a professor at Kangwon National University, South Korea, where he is a full professor. He joined Rensselaer Polytechnic Institute (Troy, USA) and University of Washington (Seattle, USA) as a visiting scholar for one year in 1993 and 2008, respectively. His interests include adaptive methods, optimization in numerical methods, and analysis on wave propagations, RF components. He is the corresponding author in this paper.

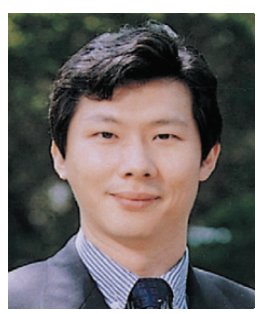

Hyeong-Seok Kim was born in Seoul, South Korea in 1962. He received his B.S., M.S., and Ph.D. degrees in electrical engineering from Seoul National University, South Korea in 1985, 1987, and 1990, respectively. From 1990 to 2002, he worked with the Division of Information Technology Engineering, Soonchunhyang University in Asan, South Korea. He was a Visiting Professor of Electrical Computer Science Engineering at the Rensselaer Polytechnic Institute in Troy, New York in 1997. He transferred to the School of Electrical and Electronics Engineering, ChungAng University, Seoul, South Korea as a professor in 2002. His current research interests include numerical analysis of electromagnetic field and waves, optimal and robust design of passive components for wireless communication, RFID applications, wireless power transmission, and electromagnetic education. 